MODELING, IDENTIFICATION AND CONTROL, 2001, VOL. 22, NO. 4, 211-225

doi:10.4173/mic.2001.4.2

\title{
Piecewise affine observer-based robust controllers for constrained nonlinear systems*
}

\author{
LARS IMSLAND $\dagger$, OLAV SLUPPHAUG $\$$ and BJARNE A. FOSS $\dagger$
}

Keywords: output feedback, robust nonlinear control, observer design, constrained systems, bilinear matrix inequalities

For a general class of constrained uncertain nonlinear discrete-time systems, and a general class of observers, it is developed bilinear matrix inequalities whose solution gives gain matrices used in a piecewise affine observer/controller structure. The closed-loop system is robustly quadratically stable with a region of attraction larger than a prescribed ellipsoidal region. It is also developed a second-order algorithm for solving these inequalities locally.

\section{Introduction}

The problem of stabilizing nonlinear systems using output measurements is a fundamental problem in control, and has received considerable attention in the literature. This is in general a hard problem, as illustrated by the fact that finding a stabilizing static output feedback (equivalent to a reduced order dynamic output feedback problem) for time-invariant linear systems is still an open problem (El Ghaoui, Oustry and AitRami, 1997; Apkarian and Tuan, 1999). Further, incorporating input and state constraints make the output feedback stabilization problem even more challenging.

In Slupphaug, Imsland and Foss (2000), the authors have attacked this problem using (reduced order) piecewise affine dynamic output feedback for constrained nonlinear discrete-time systems. The associated bilinear matrix inequality feasibility problem has proved to be hard to solve, thus motivating us to consider piecewise affine observer-based controller structures. By structuring the dynamic part of the controller this way, we are essentially limiting the space to search for controller parameters.

A common approach to the output feedback stabilization problem, is to design an observer to obtain an estimate of the state, and then (independently) design a state feedback controller using the estimated state. In the case of linear systems, using linear state feedback coupled with a linear state observer results in global asymptotic closed-loop stability as long as the observer-error dynamics and the closed-loop process under pure state feedback are asymptotically stable. In this case the separation principle is said to hold (Khalil, 1996), since the assignment of the closed loop eigenvalues can be carried out as separate tasks for the state feedback and observer problems.

For nonlinear systems, it has only been possible to establish separation principles

*This is an extended version of a paper presented at the European Control Conference 2001 in Porto, Portugal.

†Department of Engineering Cybernetics, Norwegian University of Science and Technology, 7491 Trondheim, Norway.

$\ddagger$ ABB, Process Industries Division. 
that either gives only local stability (see e.g. Scokaert, Rawlings and Meadows (1997)), or consider specific classes of systems. As an example, Atassi and Khalil (1999) shows that the performance (including asymptotic stability and region of attraction) of a globally bounded state feedback control of a certain class of nonlinear systems can be recovered using a sufficiently fast high-gain observer. Despite this, the lack of a global separation principle for general nonlinear systems suggests that designing state feedback using a state observer should in many cases be performed together, analyzing the nonlinear system and the observer as one system.

The approach taken herein is to simultaneously search for piecewise affine observerstate feedback and piecewise affine observer output injection that stabilizes the composite system, taking constraints into consideration. For a general class of uncertain nonlinear discrete-time systems, and a general class of observers, it is developed synthesis matrix inequalities (adapting a result in Slupphaug et al. (2000)) whose solution gives gain matrices used in the controller and the observer.

It is also developed a second-order algorithm for solving these matrix inequalities locally, based on an algorithm given in Fares, Apkarian and Noll (2001) for solving similar matrix inequalities. The algorithm uses general purpose LMI-solvers.

The approach is illustrated with a simple example.

\section{System and observer models}

\subsection{System description}

The system to control is a discrete time uncertain nonlinear system with an uncertain output mapping, described by

$$
\begin{gathered}
x_{k+1} \in \mathscr{F}\left(x_{k}, u_{k}, \Psi\right) \subset \mathbb{R}^{n} \\
y_{k} \in \mathscr{G}\left(x_{k}, \Psi\right) \subset \mathbb{R}^{r}
\end{gathered}
$$

where

$$
\begin{aligned}
\mathscr{F}(x, u, \Psi): & =\left\{x^{+} \mid x^{+}=f(x, u, \psi) \text { for some } \psi \in \Psi\right\} \\
\mathscr{G}(x, \Psi): & =\{y \mid y=h(x, \psi) \text { for some } \psi \in \Psi\}
\end{aligned}
$$

We will assume that the system is constrained, that is, the allowed values for the inputs and states are $u_{k} \in U \subset \mathbb{R}^{m}$ and $x_{k} \in X \subset \mathbb{R}^{n}$, respectively. We will also call these sets the model validity sets, and they contain the origin in their interiors. We assume that $f(0,0, \psi)=0$ and $h(0, \psi)=0$, thus the equilibrium input is assumed known.

Assume that the dynamics of this uncertain system can be encapsulated by a difference inclusion of local affine models, i.e.

$$
\begin{gathered}
\mathscr{F}(x, u, \Psi) \subset \mathscr{M}_{i(x)}\left(x, u, \Theta^{s}\right), \forall x \in X, u \in U \\
\mathscr{G}(x, \Psi) \subset \mathscr{H}_{i(x)}\left(x, \Theta^{s}\right), \forall x \in X
\end{gathered}
$$

Here, $i(x):=\left\{i \in I_{N_{L}} \mid x \in X_{i}^{L}\right\}$ with $I_{N}:=1, \ldots, N . X_{i}^{L}$ denotes local model validity set $i$, which is the part of the state-space in which uncertainty model $i$ is valid. The local model validity sets will without loss of generality be taken as non-overlapping (hence $i(x)$ is a singleton). Further, they shall exactly cover $X$, i.e. $X=\cup_{i \in I_{N_{L}}} X_{i}^{L} . N_{L}$ denotes 


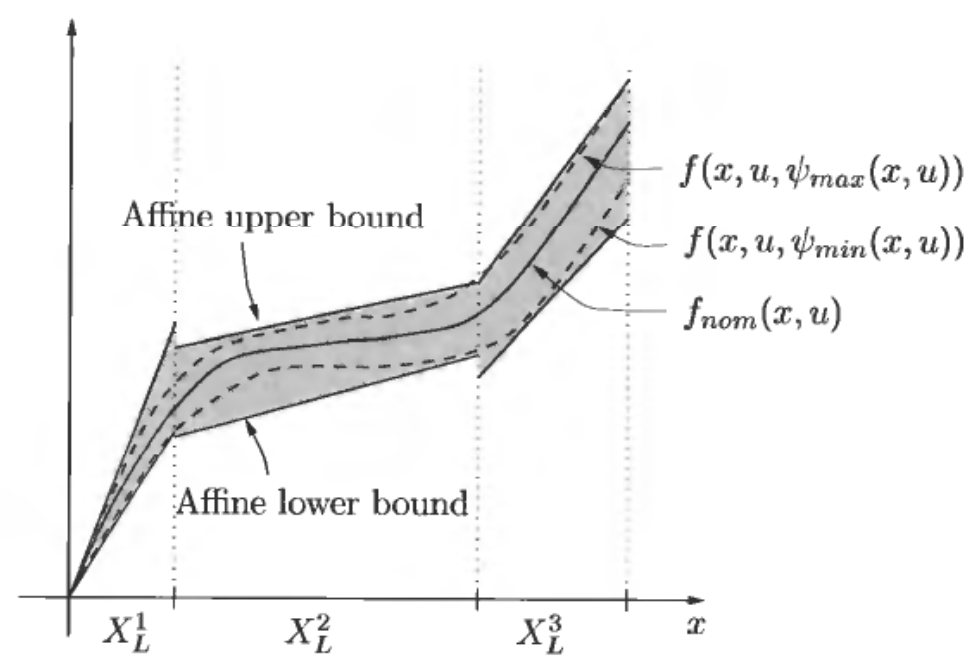

Figure 1. Illustration of the uncertainty modelling. The shaded area is accounted for in the controller synthesis.

the total number of local uncertainty models (and associated validity sets). The $\mathscr{M}_{i} \mathrm{~s}$ and $\mathscr{H}_{i}$ s are defined by

$$
\begin{aligned}
\mathscr{M}_{i}\left(x, u, \Theta^{s}\right): & =\left\{x^{+} \mid \exists \theta \in \Theta^{s}, x^{+}=A^{i}(\theta) x+B^{i}(\theta) u+c^{i}(\theta)\right\} \\
\mathscr{H}_{i}\left(x, \Theta^{s}\right): & =\left\{y \mid \exists \theta \in \Theta^{s}, y=C^{i}(\theta) x+d^{i}(\theta)\right\}
\end{aligned}
$$

where the involved matrices are affine in the parameter $\theta$, i.e. $A^{i}(\theta):=$ $A_{0}^{i}+\sum_{j=1}^{N_{\underline{\theta}}^{s}} A_{j}^{i} \theta_{j}$, and accordingly for $B^{i}(\theta), c^{i}(\theta), C^{i}(\theta)$ and $d^{i}(\theta)$. The parameter $i$ denotes which subset (local model validity set) of the state-space the matrices are valid. Further, $\theta=\left(\theta_{1}, \ldots, \theta_{N_{\theta}^{s}}\right)^{T} \in \Theta^{s}$ is the possibly time-varying parameter vector and $N_{\theta}^{s}$ denotes the number of parameters. The parameter vector is assumed to be in a hyper-rectangle

$$
\Theta^{s}:=\left\{\theta=\left(\theta_{1}, \ldots, \theta_{N_{\theta}^{s}}\right)^{T} \mid \forall j \in I_{N_{\theta}^{s}}, \theta_{j} \in[0,1]\right\}
$$

A procedure for obtaining this uncertainty description from a given nonlinear ODE with uncertain parameters is given in Slupphaug et al. (2000). If the uncertainty description is obtained by upper and lower bounding each element of the uncertain state transition map, $\mathscr{F}$, and output mapping, $\mathscr{G}$, see Figure 1 , then typically $N_{\theta}^{s}=2^{n+r}$.

For the $N_{L o}$ local model validity sets that contains the origin in their closure, the 'affine terms' $\left(c^{i}(\theta)\right.$ and $\left.d^{i}(\theta)\right)$ are identically zero. This is possible since we assumed $f(0,0, \psi)=0$ and $h(0, \psi)=0$ for all $\psi \in \Psi$.

We also define non-overlapping local output sets, $Y_{j, j}^{L}, j \in I_{M_{L}}$. These partition the output space $Y$, and are used for defining the piecewise affine observer output injection structure. By assuming that output constraints are mapped to the state space, there is no loss of generality in constructing $Y=\cup_{j \in I_{M_{L}}} Y_{j=}^{L} \mathbb{R}^{r}$. This automatically ensures that the piecewise affine observer output injection is well defined. Also define $M_{L o}$, the number of local output sets with the origin in their closure. 


\subsection{Observer description}

We assume a full state model-based observer with an 'observer correction term' $v_{k}$,

$$
\begin{gathered}
\hat{x}_{k+1}=f^{o}\left(\hat{x}_{k}, \mathrm{u}_{k}\right)+v_{k} \\
\hat{y}_{k}=g^{o}\left(\hat{x}_{k}\right)
\end{gathered}
$$

The input $u_{k} \in U$, and the state and output mappings are defined for $\hat{x}_{k} \in \hat{X} \subset R^{n}$. A natural choice for the observer model is a nominal nonlinear model of the system to be controlled.

To develop the synthesis inequalities, we will need to represent the dynamics of the observer using local affine parameter-varying models. This can be done by encapsulating the dynamics (as in Section 2.1), by an affine difference inclusion

$$
\begin{aligned}
f^{o}(\hat{x}, u) & \in \hat{\mathscr{H}}_{i(\hat{x})}\left(\hat{x}, u, \Theta^{o}\right), \forall \hat{x} \in \hat{X}, u \in U \\
g^{o}(\hat{x}) & \in \hat{\mathscr{H}}_{\hat{\imath}(\hat{x})}\left(\hat{x}, \Theta^{o}\right), \forall \hat{x} \in X
\end{aligned}
$$

Define $\hat{\imath}(\hat{x}), I_{N_{L}^{o}}, \hat{X}_{i}^{L}, \hat{A}^{i}(\theta), \Theta^{o}$ etc. as for the system, confer section 2.1 .

By encapsulating the nonlinear dynamics of the observer using this difference inclusion, we implicitly take into consideration unnecessary 'non-existing' observer dynamics (since we know the observer dynamics exactly). Depending on how many local model validity sets we choose to use, and how close the encapsulation is, this may introduce considerable conservatism.

If we choose a piecewise affine observer model,

$$
f^{o}(\hat{x}, u)=\hat{A}^{\hat{\imath}(\hat{x})} \hat{x}+\hat{B}^{i(\hat{x})} u+c^{i(\hat{x})}
$$

we can account for the exact observer dynamics. This observer model can for instance be obtained by an approximation of a nominal model. Note that an approximation can be made arbitrarily accurate by using many local model validity sets. The analysis of this structure will be a special case of the above, since it implies $\Theta^{\circ}=\emptyset$.

\section{Output feedback controller synthesis}

Based on the models above, we will develop synthesis inequalities parameterized in the matrices defining the piecewise affine observer-state feedback and observer output injection functions. Feasibility of the inequalities will imply that the feedback and observer correction structure will stabilize the given uncertainty class for the defined uncertainties.

Let $j(y)=\left\{j \in I_{M_{L}} \mid y \in Y_{j}^{L}\right\}$. When the dependence on $x$ and $y$ is understood from the context, we will write $i$ for $i(x), \hat{\imath}$ for $\hat{i}(x)$ and $j$ for $j(y)$.

\subsection{Feedback and observer injection structure}

The control $u_{k}$ and observer correction term $v_{k}$ are chosen to be affine functions of the observer state $\hat{x}_{k}$ and the output $y_{k}$,

$$
\begin{aligned}
& u_{k}=K_{s}^{\hat{i}} \hat{x}_{k}+k_{s}^{\hat{\imath}} \\
& v_{k}=K_{o}^{j}\left(y_{k}-\hat{y}_{k}\right)+k_{o}^{j}
\end{aligned}
$$

The feedback matrices $\left(K_{s}^{\hat{i}}\right.$ and $k_{s}^{\hat{i}}$ ) are chosen based on which subset $\hat{X}_{\hat{I}}^{L}$ the observer state $\hat{x}_{k}$ is in (hence superscript $\hat{\imath}=\hat{\imath}\left(x_{k}\right)$ ). The observer matrices can be based on 
which of the local output sets $Y_{j}^{L}, j \in I_{M_{L}}$, the output $y_{k}$ is in, or by choosing them based on which subset $\hat{X}_{i}^{L}$ the observer state $\hat{X}_{k}$ is in (i.e. replace $j$ with $\hat{\imath}$ ). The latter approach will typically simplify the complexity of the resulting inequalities.

For the $\hat{X}_{i}^{L} \mathrm{~s}$ and the $Y_{j}^{L}$ s containing the origin in their closure, $k_{s}^{i}$ and $k_{o}^{j}$ are zero.

Note that other feedback/observer structures that are affine in $y_{k}, \hat{x}_{k}$ and $\hat{y}_{k}$ will result in similarily structured matrix inequalities.

\subsection{Closed-loop dynamics}

Inserting the feedback and observer correction terms into the affine parameterdependent 'open-loop' equations describing the system and the observer, we obtain the closed-loop dynamics, for $x_{k} \in X_{i}^{L}, \hat{x}_{k} \in \hat{X}_{i}^{L}$ and $y_{k} \in Y_{j}^{L}$, and for some $\theta_{k}^{s} \in \Theta^{s}$ and $\theta_{k}^{o} \in \Theta^{o}, \theta_{k}:=\left[\left(\theta_{k}^{s}\right)^{T}\left(\theta_{k}^{o}\right)^{T}\right]^{T}$

$$
\left[\begin{array}{l}
x_{k+1} \\
\hat{x}_{k+1}
\end{array}\right]=\mathrm{A}\left(K_{s}^{i}, K_{o}^{j}, \theta_{k}\right)\left[\begin{array}{l}
x_{k} \\
\hat{x}_{k}
\end{array}\right]+\mathrm{c}\left(k_{s}^{\hat{i}}, K_{o}^{j}, k_{o}^{j}, \theta_{k}\right)
$$

with

$$
\begin{aligned}
\mathrm{A}\left(K_{s}^{\hat{i}}, K_{o}^{j}, \theta_{k}\right) & :=\left[\begin{array}{cc}
A^{i}\left(\theta_{k}^{s}\right) & 0 \\
0 & \hat{A}^{i}\left(\theta_{k}^{o}\right)
\end{array}\right]+\left[\begin{array}{l}
B^{i}\left(\theta_{k}^{s}\right) \\
\hat{B}^{i}\left(\theta_{k}^{o}\right)
\end{array}\right] K_{s}^{i}\left[\begin{array}{ll}
0 & I
\end{array}\right]+\left[\begin{array}{l}
0 \\
I
\end{array}\right] K_{o}^{j}\left[C^{i}\left(\theta_{k}^{s}\right)-\hat{C}^{i}\left(\theta_{k}^{o}\right)\right] \\
\mathrm{c}\left(k_{s}^{i}, K_{o}^{j}, k_{o}^{j}, \theta_{k}\right) & =\left[\begin{array}{l}
c^{i}\left(\theta_{k}^{s}\right) \\
\hat{c}^{i}\left(\theta_{k}^{o}\right)
\end{array}\right]+\left[\begin{array}{l}
B^{i}\left(\theta_{k}^{s}\right) \\
\hat{B}^{i}\left(\theta_{k}^{o}\right)
\end{array}\right] k_{s}^{i}+\left[\begin{array}{l}
0 \\
I
\end{array}\right] K_{o}^{j}\left(d^{i}\left(\theta_{k}^{s}\right)-\hat{d}^{\hat{i}}\left(\theta_{k}^{o}\right)\right)+\left[\begin{array}{l}
0 \\
I
\end{array}\right] k_{o}^{j}
\end{aligned}
$$

\subsection{The subsets of the state-space with the same observer and feedback}

For use in the stability analysis, we need to identify subsets of the total statespace where the same observer and feedback is used. We have the local model validity sets $X_{i}^{L}, i \in I_{N_{L}^{m}}$, the local observer model validity sets $\hat{X}_{\hat{i}}^{L}, \hat{\imath} \in I_{N_{L}^{v}}$ and the local output sets $Y_{j}^{L}, j \in I_{M_{L}}$, covering the state-space, $X$, observer state-space, $\hat{X}$ and output-space, $Y$, respectively. We will define subsets $X_{i i j}$ on $X \times \hat{X}$ such that, loosely speaking, the closed-loop dynamics on $X_{i i j}$ is associated with open loop dynamics $\mathscr{H}_{i}$, observer dynamics $\hat{H}_{\hat{i}}$ and observer state feedback $\hat{\imath}$ and observer output injection $j$. These subsets will be denoted intersection sets, and the union of all of them will exactly cover $X \times \hat{X}$ since $Y$ covers all the possible outputs from $X$. The intersection sets may be overlapping.

Formally, the subsets $X_{i i j}$ are given as

$$
X_{i \hat{\imath} j}:=\left(X_{i}^{L} \cap\left\{x \mid \exists \theta \in \Theta^{s} \text { s.t. } C^{i(x)}(\theta) x+d^{i(x)}(\theta) \in Y_{j}^{L}\right\}\right) \times \hat{X}_{\hat{\imath}}^{L}
$$

Note that when choosing observer matrices based on the observer state instead of the output, the desired subsets are no longer intersections, but simply defined as

$$
X_{i i}:=X_{i}^{L} \times \hat{X}_{i}^{L},
$$

which typically are much simpler to handle.

The sets defined above will form the hasis for using the $\mathscr{S}$-procedure when deriving the stability conditions for the closed-loop. 


\subsection{Set approximations}

This is done as in (Slupphaug and Foss 1999). The $X_{i f i j}$ s containing the origin in their closure are outer approximated by unbounded polytopes $\left\{x \mid E_{l} x \leqslant 0\right\}$, and are indexed with $l \in I_{N}$, the $X_{i i j} \mathrm{~s}$ not containing the origin in their closure are outer approximated either by possibly bounded polytopes $\left\{x \mid E_{l} x+e_{l} \leqslant 0\right\}$, and indexed in $\left\{N^{\mathrm{o}}+1, \ldots, N^{p}\right\}$, or by ellipsoids

$$
\left\{x \mid\left[\begin{array}{ll}
x & 1
\end{array}\right]\left[\begin{array}{ll}
E_{l} & e_{I} \\
e_{l}^{T} & \epsilon_{l}
\end{array}\right]\left[\begin{array}{l}
x \\
1
\end{array}\right] \leqslant 0\right\},
$$

indexed in $\left\{N^{p}+1, \ldots, N\right\}$. With this indexing, for each $l$ there exists a unique triple $(i, \hat{i}, j)$ which will be denoted $\left(i_{l}, \hat{l}_{l}, j_{l}\right)$, thus intersection set number $l$ is $X_{i_{i} i_{j} j_{l}}$. Note that $N^{\mathrm{o}}$ denotes the number of intersection setscontaining the origin in their closure, while $N^{p}$ is the number of intersection sets outer approximated by polytopes, and $N$ is the total number of non-empty intersection sets.

Furthermore, assume that the state-space model validity- and constraint set $X$ is inner approximated with an intersection of $N_{q x}$ ellipsoids defined by the matrices $H_{i, x}$ and the centers $x_{i, c}$. The control model validity- and constraint set $U$ is assumed inner approximated with an intersection of $N_{q u}$ ellipsoids defined by the matrices $H_{i, u}$ and the center $u_{i, c}$.

\subsection{Combined synthesis based on quadratic stability}

It is common in observer design to use the error variable $\tilde{x}_{k}=x_{k}-\hat{x}_{k}$ and design an observer for convergence of $\tilde{x}_{k}, \tilde{x}_{k} \rightarrow 0$ (i.e., $\hat{x}_{k} \rightarrow x_{k}$ ). Stability of the origin in the variables $\left[\begin{array}{cc}x_{k}^{T} & \hat{x}_{k}^{T}\end{array}\right]^{T}$ is equivalent to stability of the origin in the variables $\left[\begin{array}{ll}x_{k}^{T} & \tilde{x}_{k}^{T}\end{array}\right]^{T}$.

We want the origin of the closed-loop system to be affinely quadratically stable (Slupphaug et al., 2000), meaning that a quadratic, affinely parameter-dependent, Lyapunov function $x_{k}^{T} P\left(\theta_{k}\right) x_{k}$ exists, and that the region of attraction is larger than a given ellipsoid $R_{A}$. The affine quadratic stability of equation (9) implies that the origin of the nonlinear system equation (2) with observer based output feedback is exponentially stable.

Based on the stability definition, we can state matrix inequalities which implies that the closed-loop is affinely quadratically stable. Below, we will use $\theta$ for $\theta_{k}, \theta \in \Theta:=\Theta^{s} \times \Theta^{o} \subset \mathbb{R}^{N_{\theta}}, N_{\theta}:=N_{\theta}^{s}+N_{\theta}^{o}$.

In the following result we will need the affine functions defined by

$$
a(\theta):=a_{o}+\sum_{j=1}^{N_{\theta}} \theta_{j} a_{j}, a \in\left\{P, \alpha, W^{l}, \tau^{l}, \beta, \mu^{l}\right\} .
$$

The $W_{j}^{l}$ s are symmetric matrices whose dimension are the row dimension of the corresponding $E_{l} \mathrm{~s}$, denoted $n_{E_{i}}$. The $\alpha_{j} \mathrm{~s}, \tau_{j}^{l} \mathrm{~s}, \beta_{j} \mathrm{~s}$ and $\mu_{j}^{l} \mathrm{~s}$ are scalars.

In the theorem below,

$$
\Omega=\left[\begin{array}{ll}
I & 0 \\
I & 0
\end{array}\right] \text { or } \Omega=\left[\begin{array}{cc}
I & 0 \\
I & -I
\end{array}\right]
$$

depending on if $R_{A}$ is given in the $\left(x_{k}, \hat{x}_{k}\right)$-coordinates, or in the $\left(x_{k}, \tilde{x}_{k}\right)$-coordinates, respectively. 
Theorem 1 Let $\Theta_{0}$ be the corners of the parameter box $\Theta$, i.e. $\Theta_{0}=\{0,1\}^{N_{\theta}}$. Then, if $\exists M>0$, symmetric matrices $\left\{P_{j}\right\}_{j}^{N_{\theta}}$ and $S$, matrices of appropriate dimensions

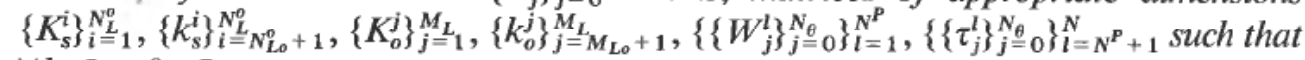
$\forall l \in I_{N^{o}}, \theta \in \Theta_{0}$

$$
\left[\begin{array}{cc}
S & \Omega A\left(K_{s}^{\hat{l}_{l}}, K_{o}^{j_{l}} ; \theta\right) \\
* & \Omega^{T} P(\theta) \Omega-M-E_{l}^{T} W^{l}(\theta) E_{l}
\end{array}\right] \geqslant 0
$$

$\forall l \in\left\{N^{o}+1, \ldots, N^{p}\right\}, \theta \in \Theta_{0}$

$$
\left[\begin{array}{ccc}
S & \Omega A\left(K_{s}^{\hat{i}_{1}}, K_{o}^{j_{l}} ; \theta\right) & \Omega \mathrm{c}\left(k_{s}^{\hat{k}_{l}}, K_{o}^{j_{l}}, k_{o}^{j_{l}} ; \theta\right) \\
* & \Omega^{T} P(\theta) \Omega-M-E_{l}^{T} W^{l}(\theta) E_{l} & -E_{l}^{T} W^{l}(\theta) e_{l} \\
* & * & -e_{l}^{T} W^{l}(\theta) e_{l}
\end{array}\right] \geqslant 0
$$

$\forall l \in\left\{N^{p}+1, \ldots, N\right\}, \theta \in \Theta_{0}$

$$
\left[\begin{array}{ccc}
S & \Omega \mathrm{A}\left(K_{s}^{\hat{i}_{l}}, K_{o}^{j_{l}} ; \theta\right) & \Omega \mathrm{c}\left(k_{s}^{\hat{i}_{l}}, K_{o}^{j_{l}}, k_{o}^{j_{l}} ; \theta\right) \\
* & \Omega^{T} P(\theta) \Omega-M-\tau^{l}(\theta) E_{l} & \tau^{l}(\theta) e_{l} \\
* & * & \tau^{l}(\theta) \varepsilon_{l}
\end{array}\right] \geqslant 0
$$

$\forall \theta \in \Theta_{0}$

$$
0<P(\theta) \leqslant S^{-1}
$$

and $\forall I \in I_{N^{p}}, \theta \in \Theta_{0}$

$$
W^{l}(\theta) \in \mathbb{R}_{E_{1}}^{n_{1}} n_{E_{1}},
$$

then the origin is an affinely quadratically stable equilibrium for the closed-loop system. If, in addition, there exist reals $\left\{\alpha_{j}\right\}_{j=0}^{N_{\theta}}$ and $\left\{\beta_{i}\right\}_{j}^{N_{0}}$ such that $\forall \theta \in \Theta_{0}$

$$
\left[\begin{array}{cc}
P(\theta)-\beta(\theta) R_{A} & 0 \\
0 & \beta(\theta)-\alpha(\theta)
\end{array}\right] \leqslant 0
$$

and reals $\left\{\left\{\mu_{j}^{l}\right\}_{j}^{N_{\theta}} 0\right\}_{l \in I_{N_{q x}}}$ such that $\forall l \in I_{N_{q x}}, \theta \in \Theta_{0}$

$$
\left[\begin{array}{cc}
\mu^{l}(\theta) H_{l, x}-\Omega^{T} P(\theta) \Omega & -\mu^{l}(\theta) H_{l, x} x_{l, c} \\
* & \mu^{l}(\theta)\left(x_{l, c}^{T} H_{l, x} x_{l, c}-1\right)+\alpha(\theta)
\end{array}\right] \leqslant 0,
$$

then the origin has a region of attraction containing $\left\{x\||| x\|_{R_{A}}^{2} \leqslant 1\right\}$ of at least $\left\{x \mid \exists \theta \in \Theta, x^{T} P(\theta) x \leqslant \alpha(\theta)\right\}$.

All $*$ are to be induced by symmetry. Note that these matrix inequalities are all LMIs, with the exception of equation (10).

The proof is very similar to the proof of Theorem 3.1 in Slupphaug et al. (2000), noting that the model has only affine terms in $\theta$, and omitted for brevity. Briefly outlined, the proof starts with the definition of affine quadratic stability by formulating the Lyapunov inequality for all the $X_{i_{i} \tilde{L}_{i}}$ s. By using the $\mathscr{S}$-procedure and the set approximations in section 3.4, these inequalities are made matrix inequalities. Further, by utilizing Schur complements, the inequalities becomes BMIs. By noting that these 
inequalities now are affine in $\theta$, feasibility for all $\theta \in \Theta$ is implied by feasibility for all $\theta \in \Theta_{0}$.

\subsection{Input constraints}

It is rather straightforward to extend Theorem 1 to take input constraints into consideration. This is done by inner approximating $U$ by intersections of ellipsoids as explained in section 3.4, and provide conditions that ensure that $u_{k}=K_{s}^{i} \hat{x}_{k}+k_{s}^{\hat{f}}$ is inside these intersections. These conditions can be posed as LMIs (Slupphaug and Foss, 1999, Slupphaug et al., 2000) that can be added to the inequalities in Theorem 1.

We have to satisfy the control constraints, $U$, in all the $\hat{X}_{i}^{L} \mathrm{~s}$. For this to be the case, it is sufficient that $\forall(p, \hat{l}) \in I_{N_{q u}} \times I_{L}^{N^{0}}, \theta \in \Theta$

$$
\left\|K_{s}^{i} \hat{x}+k_{s}^{\hat{t}}-u_{p, c}\right\|_{H_{p, u}}^{2} \leqslant 1, \hat{x} \in \hat{X}_{i}^{L} .
$$

Note that this condition is only sufficient because of the inner approximation of $U$.

When outer approximating the $\hat{X}_{i}^{L}$ s for formulating LMI conditions for satisfying control input constraints in this manner, it is only sensible to use ellipsoids, see Slupphaug and Foss (1999). Thus, we assume that ${ }^{1}$

$$
\left\{x \mid\left(\begin{array}{c}
x \\
1
\end{array}\right)^{T}\left(\begin{array}{cc}
\tilde{E}_{\hat{i}} & \tilde{e}_{\hat{i}} \\
\tilde{e}_{\hat{\imath}}^{T} & \tilde{\varepsilon}_{i}
\end{array}\right)\left(\begin{array}{c}
x \\
1
\end{array}\right) \leqslant 0\right\} \subset \hat{X}_{\hat{i}}^{L} .
$$

Using the $\mathscr{S}$-procedure and Schur complements on equation (11), we get that the LMI conditions: $\forall(p, l) \in I_{N_{q u}} \times I_{N} \exists \tau_{l, p}^{E}$

$$
\begin{aligned}
& {\left[\begin{array}{ccc}
\tau_{l, p}^{E} \tilde{E}_{l} & \tau_{l, p}^{E} \tilde{p}_{l} & \left(K_{s}^{i}\right)^{T} \\
* & 1+\tau_{l, p}^{E} \tilde{\varepsilon}_{l} & \left(k_{s}^{\hat{i}}-u_{p, c}\right)^{T} \\
* & * & H_{p, u}^{-1}
\end{array}\right] \geqslant 0} \\
& \tau_{l, p}^{E} \geqslant 0
\end{aligned}
$$

imply the sufficient condition equation (11) for satisfying control input constraints, and can be added to the LMIs in Theorem 1 . Note that these conditions imply that the constraints are satisfied for all $\hat{x}_{k} \in \hat{X}$, not only the $\hat{x}_{k}$ inside the resulting region of attraction.

\subsection{Reducing conservatism}

The synthesis inequalities guarantee a decreasing Lyapunov function in all the subsets $X_{i i j}$ of $X \times \hat{X}$. This will introduce unnecessary conservatism and computational complexity in the cases when some of the $X_{i i j}$ are outside the resulting region of attraction, since the Lyapunov decrease-condition will be ensured for subsets of no relevance for the final result. The result may be a non-succesful termination of the algorithm in the sense that no feasible solution is found. In this case we may omit subsets $X_{i \hat{i} j}$ that are outside the region of attraction. This situation is sketched

\footnotetext{
${ }^{1}$ Less conservatively, we could have used unions of ellipsoids.
} 

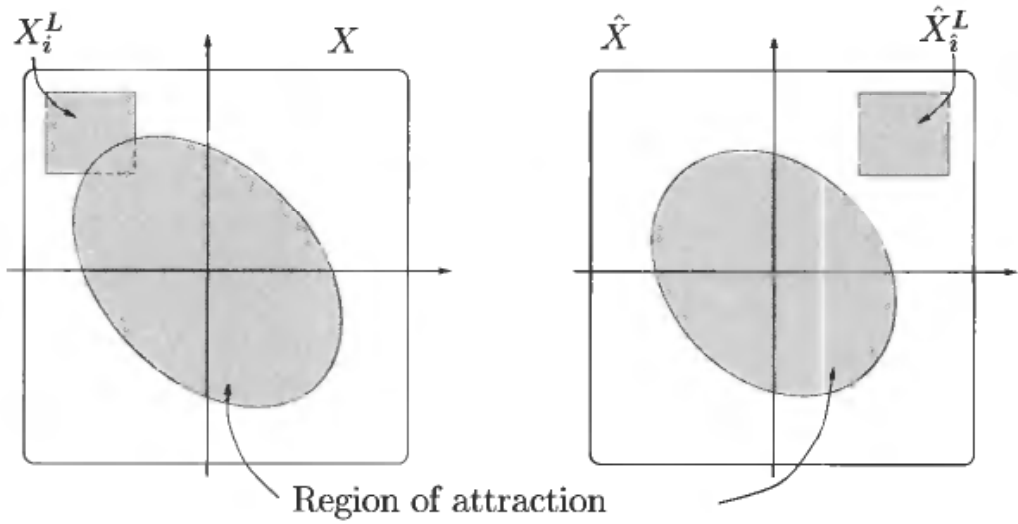

Figure 2. Illustration of one combination of $X_{i}^{L}$ and $\hat{X}_{\hat{i}}^{L}$ which can be excluded.

in Figure 2 (for simplicity, the local output sets are ignored). A practical consequence of leaving out some subsets $X_{i i j}$ is a reduction of the number of LMIs.

The argument above can be made irrespectively of which space $(X \times \hat{X}$ or $X \times \tilde{X})$ the Lyapunov function maps from. Informally, it says that we may omit LMIs that imply checking Lyapunov function decrease when the observer state is far from the real state.

Since the estimate of the region of attraction is not known in advance, one must make an assumption about the size of it and check the assumption afterwards, but $R_{A}$ will however give an indication of what it will look like. One can continue the process by reducing the size of those $X_{i i j}$ that is not expected to beentirely contained in the region of attraction.

\section{Solving the bilinear matrix inequalities}

The non-convex synthesis problem presented herein, is very similar in structure to the problem of rank minimization subject to LMI (convex) constraints, which has received substantial attention the last few years. There are reported both local and global solvers to this problem (see e.g. Apkarian and Tuan (2000)) and the references therein), and most of them have in common that they are based on efficient algorithms for solving semidefinite programming (SDP) problems (for instance, Nesterov and Nemirovskii (1994)).

The non-convex part of the synthesis inequalities presented herein, are on the form

$$
\forall \theta \in \Theta, 0<P(\theta) \leqslant S^{-1}
$$

Since $P$ is affine in $\theta$, this is equivalent to

$$
\hat{P}:=\left[\begin{array}{lll}
P\left(\theta_{1}\right) & & \\
& \ddots & \\
& & P\left(\theta_{2^{N_{\theta}}}\right)
\end{array}\right] \leqslant\left[\begin{array}{lll}
S & & \\
& \ddots & \\
& & S
\end{array}\right]^{-1}=: \hat{S}^{-1}
$$


where $\theta_{1}, \ldots, \theta_{2^{N_{\theta}}}$ are the 'corners' of the hyper-rectangle $\Theta$. This can be made an equality constraint by adding a 'slack'-variable ${ }^{2} \Delta \geqslant 0$,

$$
\hat{P}+\Delta=\hat{S}^{-1} \text {. }
$$

Having achieved this, we can adapt the method in Fares et al. (2001). They denote this method sequential semi-definite programming (SSDP), and it enjoys many similarities with sequential quadratic programming, SQP (Nocedal and Wright, 1999). The key idea is to form an augmented Lagrangian function for the BMI problem in Theorem 1:

$$
\begin{aligned}
\Phi_{c}(x, \Lambda) & =\gamma+\sum_{i j} \Lambda_{j i}((\hat{P}+\Delta) \hat{S}-I)_{i j} \\
& +\frac{c}{2} \sum_{i j}((\hat{P}+\Delta) \hat{S}-I)_{i j}^{2} \\
& =\gamma+\operatorname{tr} \Lambda((\hat{P}+\Delta) \hat{S}-I) \\
& +\frac{c}{2} \operatorname{tr}\left[((\hat{P}+\Delta) \hat{S}-I)^{T}((\hat{P}+\Delta) \hat{S}-I)\right]
\end{aligned}
$$

and minimizing this function with respect to $x=(\hat{P}, \hat{S}, \Delta, \gamma)$ subject to $x \in \chi_{L M I}$. Here, $\chi_{L M I}$ denotes a convex set given by the LMI constraints specified in Theorem 1 . The minimization is done by sequentially approximating the augmented Lagrangian function by a second-order Taylor expansion and minimizing this by solving an SDPproblem in $x$, while updating the Lagrange multiplier $\Lambda$ and the penalty parameter $c$ in a 'smart' way in each iteration.

This means that at each step $x^{(i)}$, the next step $x^{(i+1)}$ is found by use of a (e.g. backtracking) line search method (Nocedal and Wright, 1999),

$$
x^{(i+1)}=x^{(i)}+\alpha p^{(i)}
$$

where $\alpha \in[0,1]$ is a scalar stepsize, and $p^{(i)}$ is the search direction at iteration $i$. The search direction is found from the following SDP problem:

$$
\text { s.t. }\left[\begin{array}{cc}
t-\nabla \Phi_{c}(i)\left(x^{(i)}, \Lambda^{(i)}\right)^{T} p^{(i)} & \left(p^{(i)}\right)^{T} \\
p^{(i)} & \left(\nabla^{2} \Phi_{c^{(i)}}\left(x^{(i)}, \Lambda^{(i)}\right)\right)^{-1}
\end{array}\right] \geqslant 0
$$

The expressions for the gradient and Hessian are developed similarily to Fares et al. (2001). They can be found in the Appendix.

\section{Example}

Consider the uncertain constrained nonlinear system

$$
\begin{aligned}
\dot{x}_{1}(t) & =a(t) x_{1}(t)+x_{2}(t) \\
\dot{x}_{2}(t) & =\sin \left(x_{1}(t)\right)-.5 x_{2}(t)+u(t) \\
y(t) & =x_{1}(t)
\end{aligned}
$$

\footnotetext{
${ }^{2} \Delta$ should be taken as block-diagonal, with the same structure as $\hat{P}$.
} 

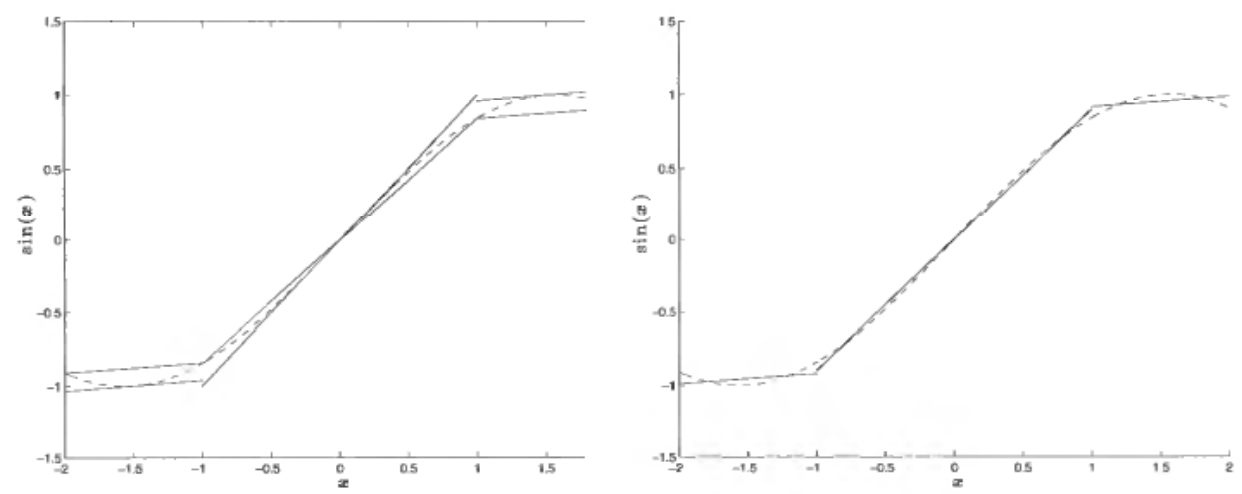

Figure 3. Piecewise affine bounding and approximation.

$$
\begin{aligned}
a(t) & \in[-.1,-.3] \\
\left|x_{1}(t)\right| & \leqslant 2,\left|x_{2}(t)\right| \leqslant 20 \\
|u(t)| & \leqslant 100
\end{aligned}
$$

which has an unstable equilibrium at the origin. The objective is to find a controller and a piecewice affine observer using the techniques developed herein that robustly stabilizes the origin for all allowed values of $a(t)$ and gives the closed loop system a region of attraction $R_{A}$ of at least $\left[\begin{array}{ll}x^{T} & \tilde{x}^{T}\end{array}\right]^{T} \in\left\{z \mid z^{T} \operatorname{diag}(1.4,12, .8,5) z \leqslant 1\right\}$. To this end, the system was discretized using forward Euler and a sample interval $h=0.01$. The nonlinearity was upper and lower bounded bypiecewise affine functions for use in the system description, while a piecewise affine approximation was used in the observer. See Figure 3.

Using the algorithm in Section 4, we found the controller and observer parameters as shown in Table 1. A phase-portrait of a simulation with this controller, is shown in Figure 4.

The control input is shown in Figure 5.

Table 1. Controller and observer matrices

\begin{tabular}{lcc}
\hline & \multicolumn{1}{c}{$u_{k}$} & $v_{k}$ \\
\hline $0 \leqslant \hat{x}_{1, k}<1$ & {$[-36.6626,-2.6169] \hat{x}_{k}$} & {$\left[\begin{array}{l}0.0738 \\
0.1603\end{array}\right] y_{k}$} \\
$-1 \leqslant \hat{x}_{1, k}<0$ & {$[-35.9716,-2.6465] \hat{x}_{k}$} & {$\left[\begin{array}{l}0.0670 \\
0.1610\end{array}\right] y_{k}$} \\
$1 \leqslant \hat{x}_{1, k} \leqslant 2$ & {$[-39.5127,-0.8942] \hat{x}_{k}+3.1701$} & {$\left[\begin{array}{l}0.1095 \\
0.2622\end{array}\right] y_{k}+\left[\begin{array}{l}-0.0101 \\
-0.0262\end{array}\right]$} \\
$-2 \leqslant \hat{x}_{1, k}<-1$ & {$[-39.6653,-0.8710] \hat{x}_{k}-3.0257$} & {$\left[\begin{array}{l}0.1127 \\
0.2719\end{array}\right] y_{k}+\left[\begin{array}{l}0.0107 \\
0.0288\end{array}\right]$}
\end{tabular}




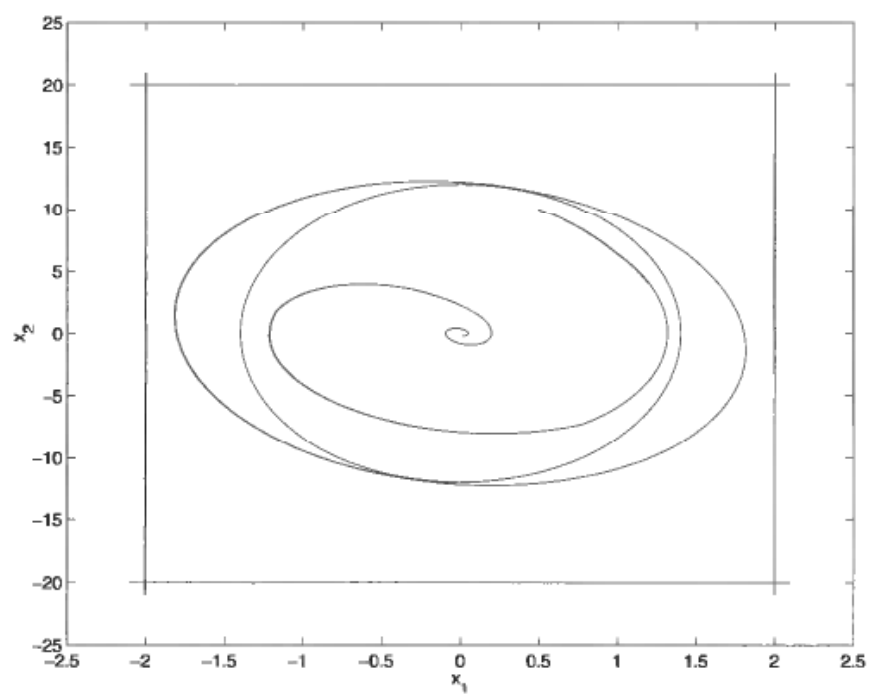

Figure 4. The state phase trajectory. The inner ellipsoid is the projection of $R_{A}$, the outer ellipsoid is the projection of an estimate of the region of attraction based on the computed Lyapunov function.

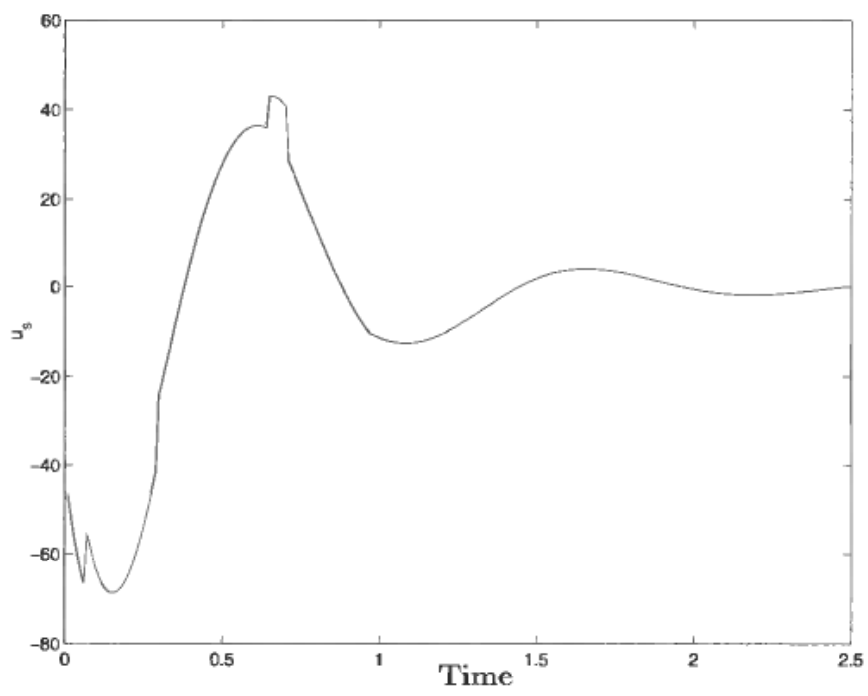

Figure 5. The input, $u_{k}$. Note that $\left|u_{k}\right| \leqslant 100$.

\section{Discussion and concluding remarks}

A mathematical programming-based approach for synthesizing observers and observer-state feedback for discrete-time constrained uncertain non-linear systems is presented. The observer-state feedback and observer output injection has a piecewise affine structure, and an estimate of the region of attraction larger than a prescribed minimum region of attraction is given as a Lyapunov level set. The region of attraction can he given in the state variables and observer-error variables, or the state variables and observer-state variables. 
A solver for the synthesis bilinear matrix inequalities is given, as an adaption of a solver presented in Fares et al. (2001). The solver is of second order, which means that it uses the (exact) Hessian of the augmented Lagrangian function. Our experience is that the solver behaves well when starting reasonably close to an optimum, and that it finds solutions to some problems where first-order solvers fail. A first-order solver based on Apkarian and Tuan (2000) is used for providing a good starting point for the second-order solver. This concave-programming based gradient-algorithm converges quickly (often only one iteration is required) to a solution relatively close to an optimum, but it requires a large number of extra LMI-variables. As is common for gradient-methods, it is prone to zigzagging when approaching the optimum.

A major issue is the growth in computational complexity that makes the presented approach prohibitive as the number of states and local model validity sets grow. If parts of the system is linear and known, however, this can be exploited and may significantly reduce the size of the feasibility problem.

The results herein can easily be specialized to the problem of designing robust asymptotic observers. This can be combined with a piecewise affine state feedback (Slupphaug et al., 2000), to give an output feedback scheme. This procedure corresponds to splitting the non-convex problem stated herein into two smaller nonconvex problems. Stability properties of this approach are discussed in Imsland, Slupphaug and Foss (2001).

\section{References}

Alizadeh, F., Haeberly, J.-P. A. and Overton, M. L. (1998). Primal-dual interior-point methods for semidefinite programming: convergence rates, stability and numerical results, SIAM J. Optim. 8(3): 746-768 (electronic).

Apkarian, P. and Tuan, H. D. (1999). Concave Programming in Control Theory, Journal of Global Optimization 15(4): 343-370.

ApKarian, P. and Tuan, H. D. (2000). Robust Control via Concave Minimization-Local and Global Algorithms, IEEE Transactions on Automatic Control 45(2): 299-305.

Atassi, A. N. and KhaliL, H. K. (1999). A separation principle for the stabilization of a class of nonlinear systems, IEEE Trans. Automat. Control 44(9): 1672-1687.

El Ghaoui, L., Oustry, F. and AitRami, M. (1997). A Cone Complementary Linearization Algorithm for Static Output-Feedback and Related Problems, IEEE Transactions on Automatic Control 42(8): 1171-6.

Fares, B., APKARIan, P. and Noll, D. (2001). An Augmented Lagrangian Method for a Class of LMI-Constrained Problems in Robust Control Theory, International Journal of Control 74(4): 348-360.

ImSLAND, L., Slupphaug, O. and Foss, B. A. (2001). Robust observer-based output feedback for nonlinear discrete-time systems with constraints, Proceedings of the 5th IFAC Nonlinear Control Systems Design Symposium (NOLCOS), St. Petersburg, Russia.

Khalil, H. K. (1996). Nonlinear Systems, 2nd edn, Prentice Hall, Upper Saddle River, NJ 07458.

Nesterov, Y. and Nemirovskil, A. (1994). Interior-point Polynomial Algorithmsin Convex Programming, vol. 13 of Studies in Applied Mathematics, SIAM, Philadelphia, PA.

Nocedal, J. and Wright, S. J. (1999). Numerical optimization, Springer-Verlag, New York.

ScokaerT. P. O. M., Rawlings, J. B. and Meadows, E. S. (1997). Discrete-time stability with perturbations: Application to model predictive control, Automatica 33(3): 463-70.

SLupPHAug, O. and Foss, B. A. (1999). Constrained Quadratic Stabilization of Discrete-Time Uncertain Nonlinear Multi-Model Systems using Piecewise Affine State-Feedback, Int. J. of Control 72(7/8): 686-701.

SluPPHAUG, O., ImSLAND, L. and Foss, B. A. (2000). Uncertainty modelling and robust output feedback control of nonlinear discrete systems: a mathematical programming approach, International Journal of Robust and Nonlinear Control 10(13): 1129-1152. 
A The gradient and Hessian of the augmented Lagrangian function

Consider $\Phi_{c}(P, S, \Delta)=\operatorname{tr}\left[\Lambda(P S+\Delta S-I)+c / 2(P S+\Delta S-I)^{T} \quad(P S+\Delta S-I)\right]$. The first derivatives can be computed to

$$
\begin{aligned}
\frac{\partial \Phi_{c}(P, S, \Delta)}{\partial \operatorname{svec} P}= & \frac{T^{2}}{2} \operatorname{svec}\left(\Lambda^{T} S+S \Lambda\right) \\
& +\frac{c}{2} T^{2} \operatorname{svec}\left((P+\Delta) S^{2}+S^{2}(P+\Delta)-2 S\right. \\
\frac{\partial \Phi_{c}(P, S, \Delta)}{\partial \operatorname{svec} S}= & \frac{T^{2}}{2} \operatorname{svec}\left(\Lambda P+P \Lambda^{T}\right)+\frac{T^{2}}{2} \operatorname{svec}\left(\Lambda \Delta+\Delta \Lambda^{T}\right) \\
& +\frac{c}{2} T^{2} \operatorname{svec}\left(S(P+\Delta)^{2}+(P+\Delta)^{2} S-2(P+\Delta)\right) \\
\frac{\partial \Phi_{c}(P, S, \Delta)}{\partial \operatorname{svec} \Delta}= & \frac{T^{2}}{2} \operatorname{svec}\left(\Lambda^{T} S+S \Lambda\right) \\
& +\frac{c}{2} T^{2} \operatorname{svec}\left((P+\Delta) S^{2}+S^{2}(P+\Delta)-2 S\right) .
\end{aligned}
$$

The second and mixed derivatives can be computed to:

$$
\begin{aligned}
\frac{\partial^{2} \Phi_{c}(P, S, \Delta)}{(\partial \mathrm{svec} P)^{2}}= & c T\left(S^{2} \circledast I\right) T \\
\frac{\partial^{2} \Phi_{c}(P, S, \Delta)}{(\partial \mathrm{svec} S)^{2}}= & c T\left((P+\Delta)^{2} \circledast I\right) T \\
\frac{\partial^{2} \Phi_{c}(P, S, \Delta)}{(\partial \mathrm{svec} \Delta)^{2}}= & c T\left(S^{2} \circledast I\right) T \\
\frac{\partial^{2} \Phi_{c}(P, S, \Delta)}{(\partial \operatorname{svec} P)(\partial \mathrm{svec} S)}= & T(\Lambda \circledast I+c(((P+\Delta) S-I) \circledast I) \\
\frac{\partial^{2} \Phi_{c}(P, S, \Delta)}{(\partial \mathrm{svec} P)(\partial \mathrm{svec} \Delta)}= & +c T\left(\left(S^{2} \circledast I\right) T\right. \\
\frac{\partial^{2} \Phi_{c}(P, S, \Delta)}{(\partial \mathrm{svec} S)(\partial \mathrm{svec} \Delta)}= & T[\Lambda \circledast I+c((S(P+\Delta)-I) \circledast I)] T \\
& +c(S \circledast(P+\Delta))] T .
\end{aligned}
$$

The symmetric Kronecker product (Alizadeh, Haeberly and Overton, 1998, Fares et al., 2001) defined by

$$
(U \circledast V) T \operatorname{svec} X=T \operatorname{svec} \frac{1}{2}\left(U X V^{T}+V X U^{T}\right),
$$

where svec is a linear operator $S^{n} \rightarrow \mathbb{R}^{n(n+1) / 2}$ defined by

$$
\operatorname{svec} X=\left[X_{11}, X_{12}, \ldots, X_{1 n}, X_{22}, X_{23}, \ldots, X_{n n}\right]^{T},
$$


basically mapping the upper right half of the symmetric input matrix into a vector. The diagonal matrix

$$
T=\operatorname{diag}[1, \sqrt{2}, \ldots, \sqrt{2}, 1, \sqrt{2}, \ldots, 1]^{T}
$$

is defined accordingly with ones on the places corresponding to the elements on the diagonal of a symmetric matrix mapped with svec, and $\sqrt{2}$ on the above-diagonal corresponding places. 